\title{
A Continuous Erector Spinae Plane Block in a Pediatric Patient: A Case Report
}

\author{
Hospital Municipal Jesus
}

\author{
Patrícia Milanez, MD, Aline Solano, MD, Pedro Paulo Vanzillotta, MD
}

\section{Introduction}

The guided ultrasound Erector Spinae Plane (ESP) block is a new, simple and safe technique of regional anesthesia ${ }^{1}$.

\section{Case Report}

A four-year-old girl $(18 \mathrm{~kg}$ ) diagnosed with empyema was admitted for a right thoracotomy. The patient had a previous INR of 1.7 therefore neuraxial techniques were avoided. General anesthesia was induced with IV lidocaine, fentanyl, propofol and rocuronium. After endotracheal intubation, the patient was placed in a left lateral position and the ESP block was performed using a high-frequency $(13-6 \mathrm{MHz})$ linear ultrasound transducer (Mturbo, Sonosite) in a longitudinal orientation, $1 \mathrm{~cm}$ lateral to the T4 spinous process. A 50-mm needle (Visioplex® - Bevel $30^{\circ}$ ) was inserted in-plane and in a cranial-to-caudal direction. The block was administered by injection of $0.4 \%$ ropivacaine $15 \mathrm{~mL}$ in total, followed by the insertion of a catheter and a continuous infusion of $0.1 \%$ ropivacaine solution combined with clonidine $1 \mathrm{mcg} / \mathrm{mL}$ at a rate of $6 \mathrm{~mL} / \mathrm{h}$ using an elastomeric pump. Additional analgesia was supplemented with dipyrone and ketoprofen.

\section{Discussion}

The ESP Block is a regional anesthesia technique recently described as a simple and safe technique ${ }^{1}$. The ultrasoundguided ESP Block involves local anesthetic injection into a paraspinal tissue plane distant from the pleura and neuraxis ${ }^{2}$. Consequently, the ESP Block can anesthetize the ventral and dorsal rami of the spinal nerve roots and the rami communicants that transmit autonomic fibers, leading to somatic and visceral analgesia.

\begin{abstract}
This method was initially described for thoracic analgesia when performed at the T5 process. Additionally, recent data indicates that at a lower vertebral level such as $\mathrm{T} 7$, it can provide effective and extensive abdominal analgesia. Finally, the relatively superficial location of the ESP Block minimizes concerns with clotting disorders ${ }^{3}$.
\end{abstract}

\section{Learning point}

To the best of our knowledge, there are only a few cases reporting a continuous ESP block in a pediatric patient. Future investigations could bring evidence to its clinical application for postoperative analgesia in pediatric anesthesia.

\section{Acknowledment}

Written informed consent was obtained from the patient's legal guardian.

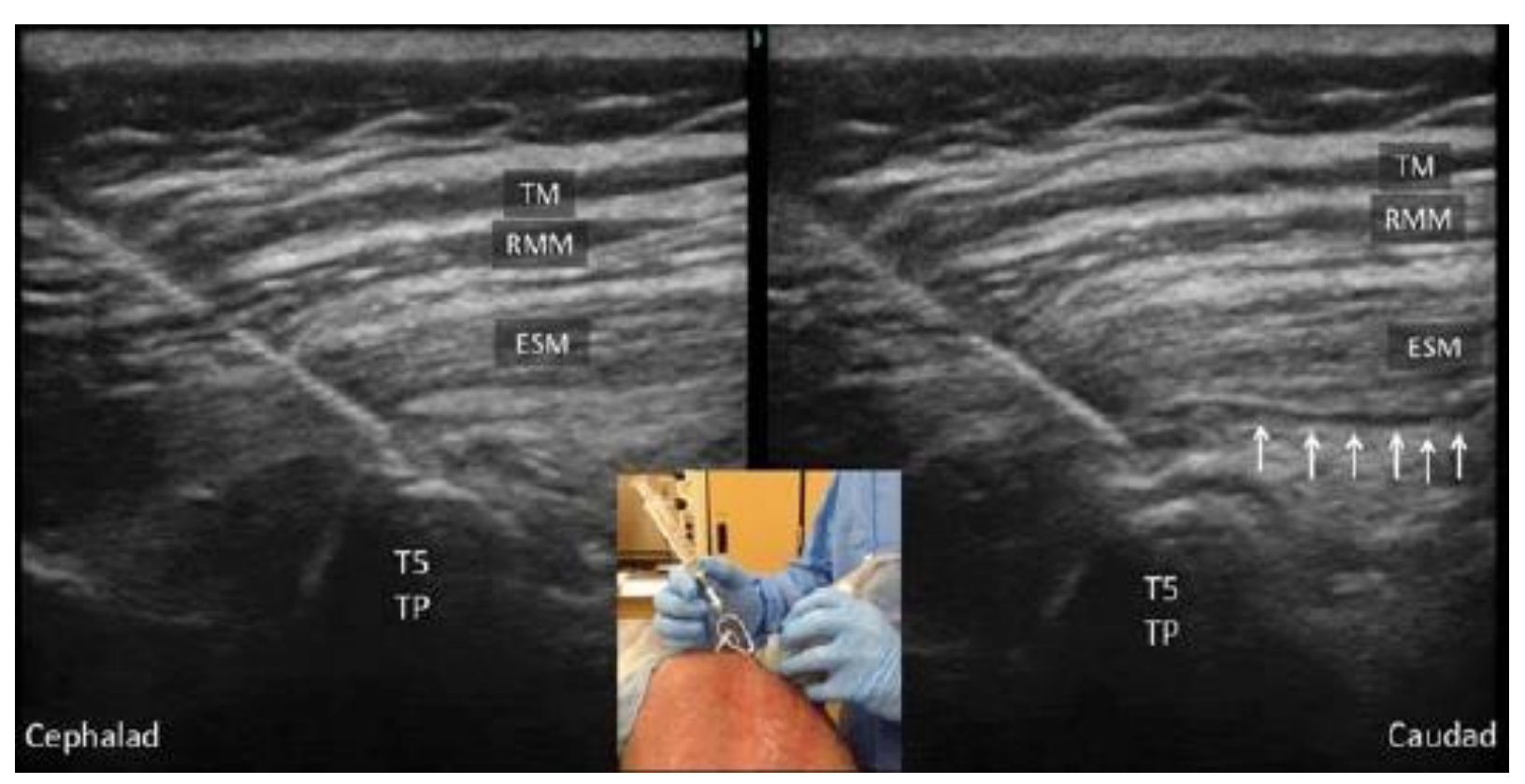

Figure 1: The Erector Spinae Block, A Novel Analgesic Technique in Thoracic Neuropathic Pain (Forero M., et al, 2016)

\footnotetext{
1 Forero M., et al: The Erector Spinae Block, A Novel Analgesic Technique in Thoracic Neuropathic Pain, Reg Anesth Pain Med.2016;41

2 Forero M., et al: The Erector Spinae (ESP) Block in the Management of Posthoracotomy Pain Syndrome, SJPain.2017; 596

${ }^{3}$ Chin J.K., et al: The Erector Spinae Block Provides Visceral Abdominal Analgesia in Bariatric Surgery, Reg Anesth Pain Med. $2017 ; 42$
} 